\title{
A Coordination Law for Multiple Air Vehicles in Distributed Communication Scenarios
}

\author{
Zhongtao Cheng, Mao Su, Lei Liu (iD, Bo Wang, and Yongji Wang \\ National Key Laboratory of Science and Technology on Multispectral Information Processing, \\ School of Artificial Intelligence and Automation, Huazhong University of Science and Technology, Wuhan, China
}

Correspondence should be addressed to Lei Liu; liulei@hust.edu.cn

Received 21 November 2019; Revised 20 May 2020; Accepted 19 June 2020; Published 4 July 2020

Academic Editor: Hocine Imine

Copyright (c) 2020 Zhongtao Cheng et al. This is an open access article distributed under the Creative Commons Attribution License, which permits unrestricted use, distribution, and reproduction in any medium, provided the original work is properly cited.

\begin{abstract}
This paper proposes a consensus-based guidance methodology for multiple air vehicles to arrive at the same spot cooperatively. First, based on the Lyapunov stability theory, a guidance law with only one control parameter is proposed, and the exact expression of total flight time can be obtained with a control parameter equal to one. Then, a two-step guidance scheme, which can achieve a finite-time consensus of the flight time, is built upon the Lyapunov-based guidance law. In the first step, on account of the information exchange between the air vehicles through an undirected and connected communication topology, a time-varying control parameter is designed to reduce the disparities of the flight time. After the consensus of the flight time, the control parameter will remain constant at one, and simultaneous arrival can be achieved. Besides, the guidance strategy is applied in a leader-follower case that one of the vehicles cannot receive information from the others and acts as the leader. The effectiveness of the proposed method is demonstrated with simulations.
\end{abstract}

\section{Introduction}

The consensus of multiple dynamic agents has a long history and can be found in many applications, such as unmanned underwater vehicles [1], mobile robots [2], sensor networks [3], and other areas. With the vigorous development of unmanned air vehicles in recent years, the simultaneous arrival of multiple air vehicles has attracted much attention. Based on information exchange between the agents, the control strategy is designed to make sure that the agents reach an agreement on the state or output values. It is obvious that simultaneous arrival can be achieved if all the agents synchronize the arrival time.

A widely used strategy to achieve simultaneous arrival is individual homing. Studies in this direction usually set a common arrival time as the desired time for each vehicle before homing, and then each vehicle tries to arrive at the target with the same specific time independently. In this way, any guidance law with the ability to control flight time can be applied to this kind of simultaneous arrival. The earliest appearance in this direction was proposed in [4]; a guidance law was proposed as a combination of two terms with the ability to control the flight time. One term was the proportion navigation guidance (PNG) law and the other was the flight time error. Then, it was applied to the simultaneous arrival case. The work in [4] was extended in [5] by controlling both arrival time and angle. The design framework in [4] was further enhanced by taking the field of view constraint into consideration in [6].

Some other studies solve the flight time control problem for simultaneous arrival with nonlinear control theory. Two-dimensional and three-dimensional guidance laws with arrival time constraint were derived using the Lyapunov stability theory in [7], where the Lyapunov candidate function contained the flight time error directly. While the work in [8] dealt with the problem indirectly, a Lyapunov candidate function concerning the heading angle error was proposed and the exact expression of the flight time was derived. However, the incomplete beta function was used and the flight time control covered only a small-time interval. 
Other studies on individual homing take advantage of the polynomial function [9-12]. In [9], the guidance command was proposed as a polynomial function with three unknown coefficients, one of which was determined to satisfy the flight time constraint. The guidance law in [10] solved the flight time control problem by following the look angle profile that was polynomial in time; both second-order and third-order polynomials were considered. The design framework in [10] was extended in [11] by considering an adaptive guidance scheme. The work in [12] generalized some preliminary solutions in this direction and extended the polynomial function to any order through mathematical induction. On the other hand, a very recent work presented a guidance scheme that can extend a certain class of existing guidance laws to satisfy the flight time constraints. The only requirement was that the time-to-go prediction was provided by the existing laws [13].

It should be noted that individual homing strategy usually requires the calculation of the achievable range of flight time for each air vehicle before homing. Then the intersection is determined from each vehicle's achievable range. A suitable common time is chosen from the intersection. It means that the cooperative arrival may fail if the intersection between each vehicle is null.

The disadvantage of the individual homing can be avoided by cooperative homing, which requires no predetermined suitable time selected from the intersection. The consensus of the flight time is achieved through communications among themselves. To the best of the author's knowledge, more interest has been directed to individual homing in the guidance literature, and rare instances of cooperative homing can be found except in [14-17], where the consensus of the vehicles' time-to-go estimates was addressed to synchronize the arrival time.

The work in [14] proposed a centralized cooperative proportion navigation (CPN) guidance law to achieve the consensus of time-to-go through a time-varying navigation gain, the calculation of which required the instantaneous time-to-go information of the air vehicle and that of all the others. The navigation gain would be updated at each time step until the instantaneous time-to-go variances went to zero. As an improvement of the work in [14], a distributed guidance law was proposed in [15], where each air vehicle merely exchanged information through an undirected and connected communication topology with its neighbors rather than all the other vehicles. Inspired by the work in [15], the research in [16] introduced a more practical case by considering both the perpendicular acceleration and the tangential acceleration, and the results were further extended to communication failure case, where one of the group vehicles cannot get information from the others. The robust guidance law proposed in [17] could still achieve simultaneous arrival without the information of faulty vehicles, which was different from the unidirectional communication error in [16]. It should be noted that the aforementioned centralized law or distributed laws achieved the cooperative arrival by the consensus of the time-to-go estimates. It should be noted that the aforementioned guidance laws are based on the consensus of the time-to-go estimation, which is accurate only toward the end of the homing process.

Motivated by the previous work, this paper proposes a consensus-based guidance law for multiple vehicles arriving at a target cooperatively. Specifically, the exact expression for total flight time can be obtained from the Lyapunov-based guidance law with control parameter equal to one. At each time step, we assume the control parameters are initialized with one, and the total flight time for each vehicle can be calculated. Then, by exchanging the total flight time between the vehicle and its neighbors under an undirected and connected communication topology, the control parameter will be adjusted to reduce the disparities of the arrival time. After the consensus of the flight time, the control parameters will remain constant at one. Furthermore, the guidance law is applied in a leader-follower case that one of the vehicles cannot receive information from the others and acts as the leader. The effectiveness of the proposed method is demonstrated with simulations. The main contributions of this paper are stated as follows:

(1) The cooperative guidance law is distributed and requires only neighboring information rather than global information, which reduces the communication burden.

(2) The previous work needs the information of the time-to-go estimates. However, the proposed guidance law deals with the consensus of the real flight time directly rather than the estimation of the timeto-go. This improves the accuracy of the guidance law.

(3) To ensure the scalability of this coordination method, the number of vehicles is not specific in the modeling and design process. Furthermore, the validity of the law is then examined in the single node failure case.

The paper is structured as follows: Lyapunov-based guidance law is introduced in Section 2. Coordination law for multiple air vehicles is offered in Section 3. Then, the guidance law is extended to a communication failure case in Section 4. Simulations are carried out in Section 5 to show the effectiveness of the proposed law. Finally, the conclusion of the work is proposed in Section 6.

\section{Lyapunov-Based Guidance Law Design}

In this paper, a scenario where multiple air vehicles arrive at a common target is considered in two-dimensional space. The planar multiple agents system is profiled in Figure 1, in which $\mathrm{X}-\mathrm{O}-\mathrm{Y}$ is an inertial reference frame denoting the vertical plane. To ensure the scalability of this coordination method, the number of vehicles, which can be hundreds or more, is not specific in the modeling and design process.

Considering that the communication scenarios can be very complex for a group of vehicles, communication state between different vehicles is defined as a binary variable, and communication topology between the air vehicles is denoted by $\mathbf{G}(E, A)$. Before moving on, it is necessary to present 


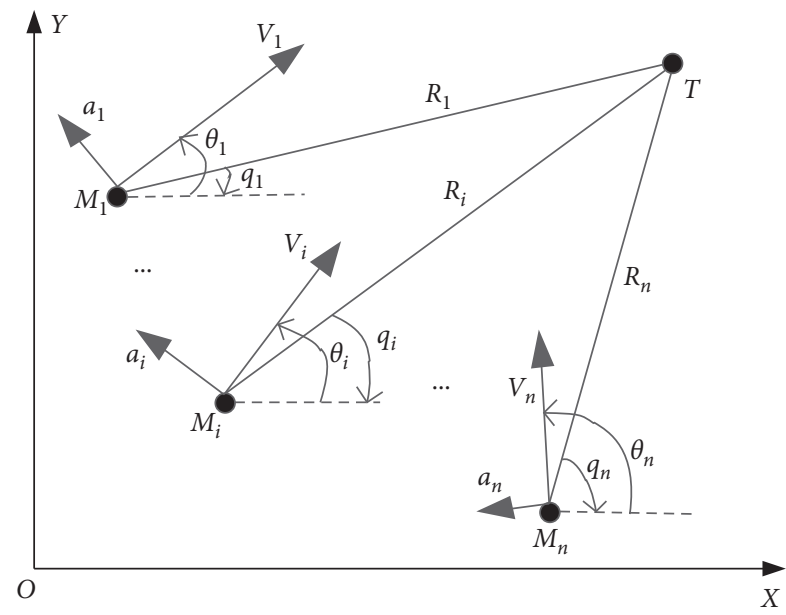

Figure 1: Engagement geometry.

some basic fundamental facts. $E$ stands for the set of edges, edge $(i, j)$ means that $i^{\text {th }}$ vehicle and $j^{\text {th }}$ vehicle are neighbors, and $j^{\text {th }}$ vehicle can receive information from $i^{\text {th }}$ vehicle. A graph is called undirected if, for any $(i, j) \in E$, $(j, i) \in E$. An undirected graph is called connected if there is an undirected path between any two different vehicles. $\mathbf{A}=$ $\left[a_{i j}\right]_{N \times N}$ is the adjacency matrix, $a_{i j}=1$ if the $i^{\text {th }}$ vehicle can get information from the $j^{\text {th }}$ vehicle, and $a_{i j}=0$ if it cannot. Besides, the Laplacian matrix $\mathbf{L}=\left[l_{i j}\right]_{N \times N}$ of $\mathbf{G}$ associated with adjacency matrix $\mathbf{A}$ is defined as $l_{i j}=-a_{i j}, i \neq j$ and $l_{i j}=\sum_{j=1}^{N} a_{i j}, i=j$.

The following assumptions are claimed before deriving the kinematic equations. First, the target is assumed to be stationary. Second, the speed of each air vehicle remains constant during the process but may not be the same as that of other vehicles. Third, the communication topology $\mathbf{G}$ of the multiagents system is assumed to be undirected and connected. The aforementioned assumptions can lead to the following lemmas.

Lemma $\mathbf{1}$ (see [18]). One eigenvalue of $\mathbf{L}$ is zero, with $\mathbf{1}$ being the right eigenvector. It can be expressed mathematically as $\mathbf{L} 1=0 \mathbf{1}$, where $\mathbf{1}$ denotes a column vector with all entries equal to one. Moreover, all nonzero eigenvalues have positive real parts.

Lemma 2 (see [19]). $x^{T} \mathbf{L} x \geq \lambda x^{T} x$ if $x$ satisfies $\mathbf{1}^{T} x=0$, where $x$ refers to any $x \in \mathbf{R}^{n}$ and $\lambda$ denotes the smallest nonzero value of the Laplacian matrix $\mathbf{L}$.

In Figure 1, a subscript $i$ is added to demonstrate variables associated with the $i^{\text {th }}$ vehicle. $\theta$ denotes the heading angle, which is the angle between the velocity vector and the fixed reference axis. $q$ denotes the line of sight (LOS) angle. The heading error angle $\sigma$ is the angle between the velocity vector and LOS vector. All the angles are measured counterclockwise. The relationship between the aforementioned angles is

$$
\sigma_{i}=\theta_{i}+\left(-q_{i}\right)=\theta_{i}-q_{i}, \quad i=1, \ldots, n .
$$

We can obtain the two-dimensional kinematic equations from the engagement geometry as

$$
\begin{aligned}
& \dot{x}_{i}=V_{i} \cos \theta_{i}, \quad i=1, \ldots, n, \\
& \dot{y}_{i}=V_{i} \sin \theta_{i}, \quad i=1, \ldots, n,
\end{aligned}
$$

where $x$ and $y$ denote the instantaneous positions of the air vehicle. The heading angle turning rate is connected with the lateral acceleration $a$ by

$$
\dot{\theta}_{i}=\frac{a_{i}}{V_{i}}, \quad i=1, \ldots, n,
$$

and $R$ denotes the relative range between target and vehicle. The differential equations for the relative range and LOS angle are

$$
\begin{aligned}
& \dot{R}_{i}=-V_{i} \cos \sigma_{i}, \quad i=1, \ldots, n, \\
& \dot{q}_{i}=\frac{-V_{i} \sin \sigma_{i}}{R_{i}}, \quad i=1, \ldots, n .
\end{aligned}
$$

In order to arrive at the target with zero miss distance, the velocity vector should aim directly at the target, which means the heading error angle should reach zero before or at the instant of arrival. Considering this, the following Lyapunov candidate function is proposed:

$$
W_{i}=2 \sin ^{2} \frac{\sigma_{i}}{2}, \quad i=1, \ldots, n .
$$

The derivative of $W$ with respect to time is

$$
\dot{W}_{i}=\sin \sigma_{i} \cdot \dot{\sigma}_{i}, \quad i=1, \ldots, n .
$$

To make each vehicle satisfy the Lyapunov asymptotic stability condition, the heading error rate is proposed as

$$
\dot{\sigma}_{i}=-\frac{c_{i} V_{i}}{R_{i}} \sin \sigma_{i}, \quad c_{i}>0, i=1, \ldots, n,
$$

where $c$ is the control parameter for each vehicle. Substituting equation (9) into equation (8), we have

$$
\dot{W}_{i}=-\frac{c_{i} V_{i}}{R_{i}} \sin ^{2} \sigma_{i}, \quad i=1, \ldots, n .
$$

It is obvious that $\dot{W}$ will be negative definite if $c>0$. Besides, equation (7) implies that $W$ is positive definite. Hence, the Lyapunov asymptotic stability condition can be met under the proposed law.

Dividing equations (9) and (5) side by side yields

$$
\frac{\mathrm{d} \sigma_{i}}{\tan \sigma_{i}}=\frac{c_{i}}{R_{i}} \mathrm{~d} R_{i}, \quad i=1, \ldots, n .
$$

Integrating both sides of equation (11), we have

$$
\sin \sigma_{i}=\left(\frac{R_{i}}{R_{0 i}}\right)^{c_{i}} \sin \sigma_{0_{i}}, \quad i=1, \ldots, n .
$$

Equation (12) illustrates that the heading error is connected with the relative range, the value of which will decline to zero as engagement proceeds. In the meantime, equation (5) indicates that the relative range will decrease monotonously. This signifies that the value of the heading error will also converge to zero at the end of the flight. 
Combining equations (12) and (9) yields

$$
\dot{\sigma}_{i}=-\frac{c_{i} V_{i}}{R_{0 i}^{c_{i}}}\left(R_{i}\right)^{c_{i}-1} \sin \sigma_{0 i}, \quad i=1, \ldots, n .
$$

According to equation (13), control value $c<1$ will inevitably lead to an undesirable situation as the relative range goes to zero in the terminal guidance situation. This valuable information indicates that it is necessary to require $c \geq 1$ in the terminal guidance situation.

Since the proposed guidance law can also be used in a midcourse guidance situation, the relative range of which will not go near zero, there is no need to worry about the undesired situation caused by zero relative range. Then the value of the control parameter just needs to meet the requirement of the Lyapunov asymptotic stability condition, which is $c>0$. So the reasonable range for the control parameter is

$$
\left\{\begin{array}{l}
c_{i}>0 \text { midcourse guidance, } \quad i=1, \ldots, n, \\
c_{i} \geq 1 \text { terminal course guidance, } \quad i=1, \ldots, n .
\end{array}\right.
$$

Differentiating equation (1) with respect to time results in

$$
\dot{\sigma}_{i}=\dot{\theta}_{i}-\dot{q}_{i}, \quad i=1, \ldots, n .
$$

Substituting equations (6) and (9) into equation (15), we get

$$
\dot{\theta}_{i}=-\left(c_{i}+1\right) \frac{V_{i}}{R_{i}} \sin \sigma_{i}, \quad i=1, \ldots, n .
$$

The following guidance command can be obtained from equations (4) and (16):

$$
a_{i}=-\left(c_{i}+1\right) \frac{V_{1}^{2}}{R_{i}} \sin \sigma_{i}, \quad i=1, \ldots, n .
$$

Substituting equation (12) into equation (17) yields

$$
a_{i}=-\left(c_{i}+1\right) \frac{V_{i}^{2}}{R_{0 i}^{c_{i}}} R^{c_{i}-1} \sin \sigma_{0 i}, \quad i=1, \ldots, n .
$$

Suppose that the control parameter $c$ for the vehicles is fixed at one, and we are going to see the flight time calculation under this specific circumstance.

Substituting $c=1$ into equation (13) yields

$$
\dot{\sigma}_{i}=-\frac{V_{i}}{R_{0 i}} \sin \sigma_{0 i}, \quad i=1, \ldots, n
$$

From equation (19), we know that the heading error rate remains negative, meaning that heading error will decrease monotonously. From equation (12), we know that the heading error will go to zero with relative range. As a result, the heading error will decrease from the initial value all along to zero at the end of the flight. Furthermore, equation (19) also implies that the heading error rate is constant. Hence, dividing the total variation of the heading error by its change rate, the analytical form of the total flight time can be acquired as

$$
t_{i}=\frac{0-\sigma_{0 i}}{\dot{\sigma}_{i}}=\frac{\sigma_{0 i} R_{0 i}}{V_{i} \sin \sigma_{0 i}}, \quad i=1, \ldots, n .
$$

If every vehicle's total flight time calculated from equation (20) is equal to the others, simultaneous arrival can be achieved. Then the main objective of this paper is to find a guidance law to reduce the flight time disparities between different vehicles.

Remark 1. The proposed Lyapunov-based guidance law can achieve the basic objective of reducing the relative distance to an acceptable order of magnitude. Utilizing of the Lyapunov stability condition can make sure that the system is stable.

Remark 2. Equation (20) gives the mathematical expression of the flight time with the vehicle's initial condition. The exact mathematical expression of the vehicles' total flight time can be derived if the control parameter equals one. No estimation or linearization is used in the process.

\section{Coordination Law for Multiple Air Vehicles}

3.1. Design Strategy. Enlightened by the mathematical acquisition of the total flight time in equation (20), a two-step control strategy is proposed here to achieve the cooperative guidance law.

First, assume that all the vehicles are under the proposed Lyapunov-based guidance law with control parameter equal to one, such that equation (20) can be used to calculate the total flight time once the initial conditions are given. Then, each time step is viewed as the initial time and the instantaneous states are treated as the initial states; use equation (20) to recalculate the flight time. Then equation (20) should be updated accordingly:

$$
t_{i}^{\prime}=\frac{\sigma_{i} R_{i}}{V_{i} \sin \sigma_{i}}, \quad i=1, \ldots, n .
$$

It is obvious that the flight time calculated from equation (21) can also be viewed as the real time-to-go. The total flight time can be written as

$$
t_{i}=t+t_{i}^{\prime}, \quad i=1, \ldots, n
$$

where $t$ is the instantaneous flight time for the vehicles. We choose the vehicle's total flight time as the consensus variable. The consensus error of the vehicles' total flight time under the undirected and connected communication topology is defined as

$$
\varepsilon_{i}=\sum_{j=1}^{n} a_{i j}\left(t_{j}-t_{i}\right), \quad i=1, \ldots, n .
$$

Second, adjust the control parameter to make the total flight time reach an agreement. In the previous discussion, we know that the consensus of the total flight time for the vehicles can lead to a simultaneous arrival. Once the consensus error calculated from equation (23) is zero, the control parameter for all the vehicles will change to one and remain there.

3.2. Coordination Law. Substituting equation (22) into equation (23) yields 


$$
\varepsilon_{i}=\sum_{j=1}^{n} a_{i j}\left(t_{j}^{\prime}-t_{i}^{\prime}\right), \quad i=1, \ldots, n .
$$

The analytical form of the flight time in equation (20) is derived from dividing the total variation of the heading error by its changing rate. In order to achieve the consensus of the flight time, the vehicles with larger flight time should increase their heading error changing rate, while the others with smaller flight time decrease their changing rate to delay the flight time. Based on the information exchange between vehicles via the communication network, the heading error in the first step is proposed as

$$
\dot{\sigma}_{i}=-\left(1-k_{i}\left|\varepsilon_{i}\right|^{u} \operatorname{sgn}\left(\varepsilon_{i}\right)\right) \frac{V_{i} \sin \sigma_{i}}{R_{i}}, \quad i=1, \ldots, n,
$$

where $u$ is a constant that satisfies $0<u<1$. It is obvious that the proposed heading error rate is under the Lyapunovbased guidance law structure, where the control parameter for each vehicle is $c_{i}=1-\left|\varepsilon_{i}\right|^{u} \operatorname{sgn}\left(\varepsilon_{i}\right)$. Once the total flight time arrives at a consensus, the control parameter for each vehicle will be fixed at one.

Before moving on, two other lemmas are introduced in advance.

Lemma 3 (see [20]). For $x_{i} \in R, i=1, \ldots, n, 0<a \leq 1$; then,

$$
\sum_{i=1}^{n}\left|x_{i}\right|^{a} \geq\left(\sum_{i=1}^{n}\left|x_{i}\right|\right)^{a}
$$

Lemma 4 (see [21]). If there exists a Lyapunov function $V(x)$ such that

$$
\dot{V}(x) \leq-a V^{m}(x),
$$

where $a>0$ and $0<m<1$, then $V(x)$ will converge to zero or a small neighborhood of zero before the final time. The settling time $T$, depending on initial condition state $x_{0}$, is given by

$$
T \leq \frac{V\left(x_{0}\right)^{1-m}}{a(1-\lambda)} .
$$

Theorem 1. The proposed heading error rate in equation (25) can make $\varepsilon_{i}, i=1, \ldots, n$ converge to zero in finite time, and the simultaneous arrival problem for the multivehicles system in Section 2 can be solved.

Proof. Differentiating equation (21) with respect to time yields

$$
\dot{t}_{i}^{\prime}=\frac{\dot{\sigma}_{i} R_{i}}{V \sin \sigma_{i}}+\frac{\sigma_{i} \dot{R}_{i}}{V_{i} \sin \sigma_{i}}-\frac{\dot{\sigma}_{i} \sigma_{i} R_{i} \cos \sigma_{i}}{V_{i} \sin \sigma_{i}}, \quad i=1, \ldots, n .
$$

Substituting equation (25) into (29), we have

$$
\begin{aligned}
\dot{t}_{i}^{\prime}= & \left(k_{i}\left|\varepsilon_{i}\right|^{u} \operatorname{sgn}\left(\varepsilon_{i}\right)-1\right)-\frac{\sigma_{i} \cos \sigma_{i}}{\sin \sigma_{i}} \\
& -\frac{\left(k_{i}\left|\varepsilon_{i}\right|^{u} \operatorname{sgn}\left(\varepsilon_{i}\right)-1\right) \sigma_{i} \cos \sigma_{i}}{\sin \sigma_{i}}, \quad i=1, \ldots, n,
\end{aligned}
$$

where $\sigma_{i}$ are usually small angles; then, $\sin \sigma_{i} \approx \sigma_{i}$ and $\cos \sigma_{i} \approx 1-\sigma_{i}^{2} / 2$. Hence, equation (30) can be rewritten as

$$
\dot{t}_{i}^{\prime}=\left(1-\cos \sigma_{i}\right) k_{i}\left|\varepsilon_{i}\right|^{u} \operatorname{sgn}\left(\varepsilon_{i}\right), \quad i=1, \ldots, n .
$$

The following Lyapunov candidate function is considered:

$$
V_{1}=\frac{1}{2} \sum_{i, j \in} a_{i j}\left(t_{j}^{\prime}-t_{i}^{\prime}\right)^{2}=\frac{1}{2} t^{T} \mathbf{L} t
$$

where $t=\left[t_{1}, \ldots, t_{n}\right]$. The derivative of $V_{1}$ with respect to time is given by

$$
\dot{V}_{1}=\dot{t}^{T} \mathbf{L} t=-\sum_{i=1}^{n}\left(1-\cos \sigma_{i}\right) k_{i}\left|\varepsilon_{i}\right|^{u} .
$$

Note that the last equality in equation (33) is obtained by using the fact that $\mathbf{L} 1=0$ and $\varepsilon=-\mathbf{L} t$. Define

$$
k=\min \left(1-\cos \sigma_{i}\right) k_{i}, \quad i=1, \ldots, n .
$$

Then, we have

$$
\dot{V}_{1} \leq-k \sum_{i=1}^{n}\left|\varepsilon_{i}\right|^{u+1} \leq-k\left(\varepsilon^{T} \varepsilon\right)^{u+1 / 2}
$$

As $\mathbf{1}^{T} \mathbf{L} 1=0=\left(\mathbf{L}^{1 / 2} 1\right)^{T}\left(\mathbf{L}^{1 / 2} 1\right)$, we can get $\mathbf{L}^{1 / 2} \mathbf{1}=0$. Then we have $\mathbf{1}^{T} \mathbf{L}^{1 / 2} t=0$. According to Lemma 1, we can get $t^{T} \mathbf{L} \mathbf{L} t \geq \lambda t^{T} \mathbf{L} t$, which can be written as $\varepsilon^{T} \varepsilon \geq 2 \lambda V$. On account of these analyses, the following equation can be driven from equation (35):

$$
\dot{V}_{1} \leq-k(2 \lambda)^{1+u / 2} V_{1}^{1+u / 2} .
$$

According to finite-time convergence theory from Lemma $4, V_{1}$ will converge to zero or a small neighbor of zero in finite time. The convergence of $V_{1}$ also means that the consensus error $\varepsilon_{i}$ will converge to zero. Once the consensus error reaches zero, the simultaneous arrival can be achieved. In addition, the consensus time is given by

$$
T \leq \frac{2 V_{1}^{(1-u / 2)}}{k(1-u)(2 \lambda)^{1-u / 2}},
$$

which completes the proof of Theorem 1.

Remark 3. Different from previous works [14-17], where the consensus of the time-to-go estimations is considered, this paper deals with the consensus of the flight time directly. Moreover, the assumption that $r_{i}>0$ and $\sigma_{i} \neq 0$ before the consensus is not necessary. Thus, the guidance law is more operationally effective. Compared with $[14,15]$, only the neighboring information is required rather than the global information in this method. Hence, the guidance law is distributed. 


\section{Extension to a Communication Failure Case}

In this subsection, the communication faults scenario that one of the group vehicles cannot receive information from other vehicles is considered. As a result, the flight time for this fault vehicle cannot be adjusted. The only way to make cooperative arrival possible in this case is that all the other vehicles coordinate their flight time with the fault one, which will be viewed as the leader.

The communication topology is viewed as a leaderfollower graph $\mathbf{G}^{\prime}$ with the fault vehicle as the root, which will be denoted as the $n^{\text {th }}$ one. In this case, the control parameter for the $n^{\text {th }}$ vehicle will remain constant at one.

With the assumption in this section, the Laplacian matrix of $\mathbf{G}^{\prime}$ can be denoted as

$$
\mathbf{L}=\left[\begin{array}{cc}
\mathbf{L}_{1} & \mathbf{L}_{2} \\
0_{1 \times(n-1)} & 0
\end{array}\right],
$$

where $\mathbf{L}_{1} \in R^{(n-1) \times(n-1)}$ is symmetric and $\mathbf{L}_{2} \in R^{n-1}$. It is obvious that

$$
\mathbf{L}_{1} \mathbf{1}=-\mathbf{L}_{2}
$$

Theorem 2. The proposed heading error rate in equation (25) can solve the simultaneous arrival problem for the multivehicles system when the communication topology is G'.

Proof. Let $\tilde{t}=\left[t_{1}, \ldots, t_{n-1}\right]^{T}$; the Lyapunov candidate function is proposed as

$$
V_{2}=\frac{1}{2}\left(\tilde{t}-\tilde{t}_{n} \mathbf{1}\right)^{T} \mathbf{L}_{1}\left(\tilde{t}-\tilde{t}_{n} \mathbf{1}\right)
$$

It can be concluded from Lemma 1 that $\mathbf{L}_{1}$ is positive definite. Let $\widetilde{\mathcal{\varepsilon}}=\left[\varepsilon_{1}, \ldots, \varepsilon_{n-1}\right]^{T}$; we have

$$
\widetilde{\varepsilon}=-\left[\begin{array}{ll}
\mathbf{L}_{1} & \mathbf{L}_{2}
\end{array}\right]\left[\begin{array}{ll}
\widetilde{t} & t_{n}
\end{array}\right]^{T} \text {. }
$$

Combining equations (39) and (41) yields

$$
\widetilde{\varepsilon}=-\mathbf{L}_{1}\left(\widetilde{t}-t_{n} \mathbf{1}\right) \text {. }
$$
have

Differentiating equation (40) with respect to time, we

$$
\begin{aligned}
\dot{V}_{2} & =\left(\widetilde{t}-\tilde{t}_{n} \mathbf{1}\right)^{T} \mathbf{L}_{1}\left(\dot{\tilde{t}}-\dot{\tilde{t}}_{n} \mathbf{1}\right) \\
& =-\sum_{i=1}^{n-1}\left(1-\cos \sigma_{i}\right) k_{i}\left|\varepsilon_{i}\right|^{u} .
\end{aligned}
$$

Similar to the proof Theorem 1, the following equation can be driven:

$$
\dot{V}_{2} \leq-\widetilde{k}\left(\widetilde{\mathcal{\varepsilon}}^{T} \widetilde{\mathcal{\varepsilon}}\right)^{u+1 / 2}
$$

where

$$
\widetilde{k}=\min \left(1-\cos \sigma_{i}\right) k_{i}, \quad i=1, \ldots, n-1 .
$$

Note that

$$
\left(\mathbf{L}_{1}^{1 / 2}\left(\widetilde{t}-\widetilde{t}_{n} \mathbf{1}\right)\right)^{T} \mathbf{L}_{1}\left(\mathbf{L}_{1}^{1 / 2}\left(\widetilde{t}-\widetilde{t}_{n} \mathbf{1}\right)\right) \geq \lambda\left(\mathbf{L}_{1}^{1 / 2}\left(\widetilde{t}-\widetilde{t}_{n} \mathbf{1}\right)\right)^{T}\left(\mathbf{L}_{1}^{1 / 2}\left(\widetilde{t}-\widetilde{t}_{n} \mathbf{1}\right)\right),
$$

which means that $\widetilde{\varepsilon}^{T} \widetilde{\mathcal{\varepsilon}} \geq 2 \lambda V_{2}$. On account of these analyses, the following equation can be driven from equation (35):

$$
\dot{V}_{2} \leq-\tilde{k}(2 \lambda)^{1+u / 2} V_{2}^{1+u / 2}
$$

According to finite-time convergence theory from Lemma $4, V_{2}$ will converge to zero or a small neighbor of zero in finite time. The convergence of $V_{2}$ also means that the consensus error $\varepsilon_{i}$ will converge to zero, and the simultaneous arrival can be achieved. Hence, Theorem 2 has been proven.

\section{Simulations}

In this section, numerical simulations are carried out to show the effectiveness of the proposed strategies. The simulation step is $0.01 \mathrm{~s}$. All the simulations are terminated when the sign of the relative velocity becomes positive or the relative range is less than $0.01 \mathrm{~m}$. We consider four vehicles arriving at a common target from different directions, and the target is fixed at $(8000,0) \mathrm{m}$. Detailed simulation parameters for the vehicles are tabulated in Table 1.

5.1. Case 1. Undirected and Connected. In this subsection, simulations are carried out to show the effectiveness of the proposed law under undirected and connected communication topology, which is demonstrated in Figure 2. The detailed simulation parameters are tabulated in Table 1.

An undirected path exists between any two different vehicles. Hence, all the vehicles can receive information from their neighbors. The Laplacian matrix of the communication topology can be acquired as

$$
\mathbf{L}=\left[\begin{array}{cccc}
3 & -1 & -1 & -1 \\
-1 & 2 & -1 & 0 \\
-1 & -1 & 3 & -1 \\
-1 & 0 & -1 & 3
\end{array}\right]
$$

Simulation results are demonstrated in Figure 3. Solid line, dashed line, dash-dotted line, and dotted line stand for the results of vehicles $1,2,3$, and 4, respectively. Combining the vehicle trajectories in Figure 3(a) and range variation in Figure 3(d), we can see that simultaneous arrival can be achieved under the proposed guidance law. The variance of the heading error angles is in Figure 3(b), all of which decline to zero at the end of the engagement, which verifies the analysis in equation (12). The consensus error of the flight time is demonstrated in Figure 3(e). It is obvious that the flight time of each vehicle can reach an agreement in finite time under the proposed law. Once the consensus of flight time is achieved, the control parameter will remain constant at 1 . We know that the acceleration will remain constant if the control parameter remains at 1 , which is consistent with the simulation results in Figure 3(c). This simulation proves 
TABle 1: Initial parameters for the four vehicles.

\begin{tabular}{lcccc}
\hline Vehicle & Initial relative range $(\mathrm{m})$ & Velocity $(\mathrm{m} / \mathrm{s})$ & Initial heading angle (deg.) & Initial LOS angle (deg.) \\
\hline 1 & 8000 & 270 & 60 & 0 \\
2 & 7500 & 250 & 30 & 0 \\
3 & 7700 & 220 & 45 & 0 \\
4 & 7000 & 200 & 30 & 0 \\
\hline
\end{tabular}

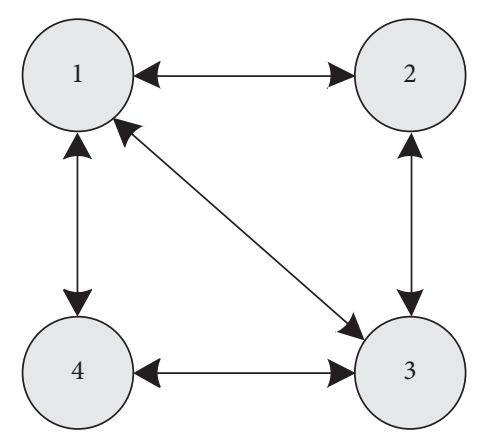

Figure 2: Undirected and connected communication topology among vehicles.
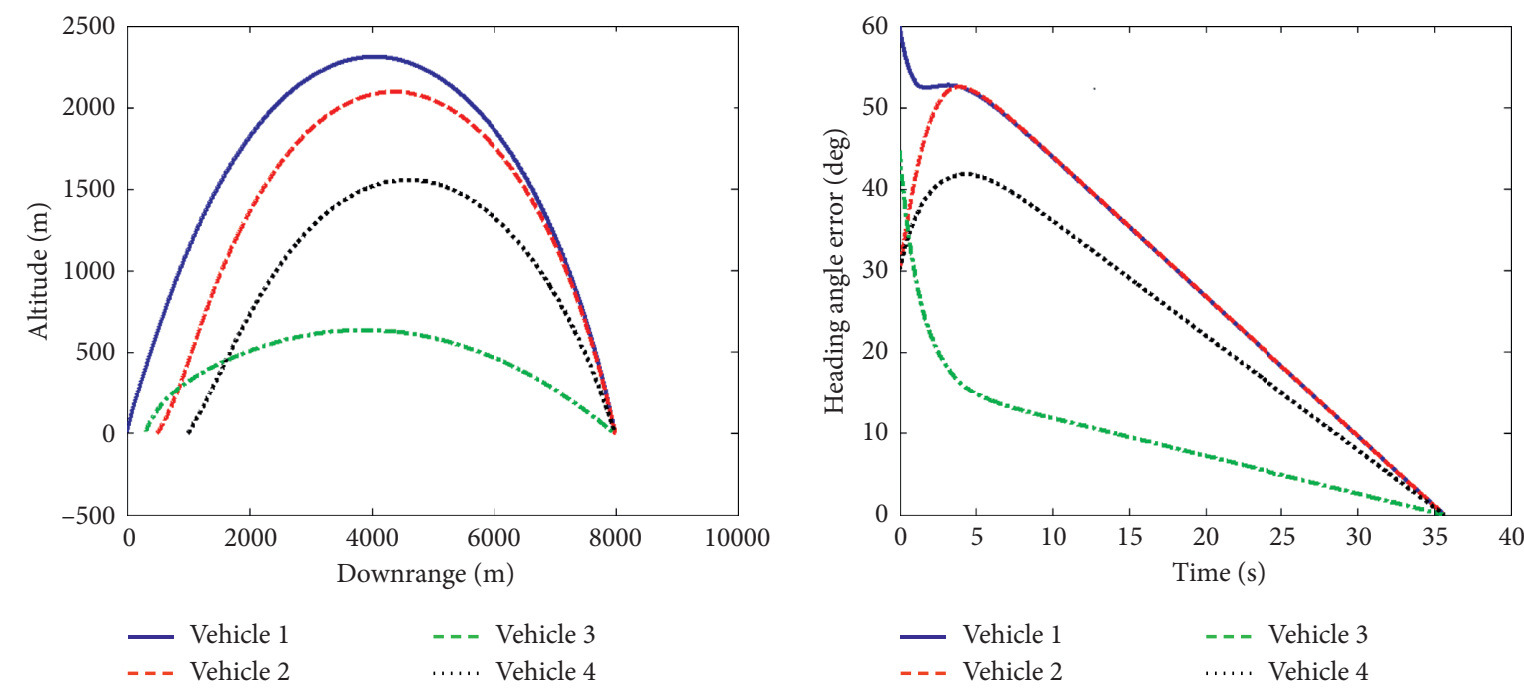

(a)

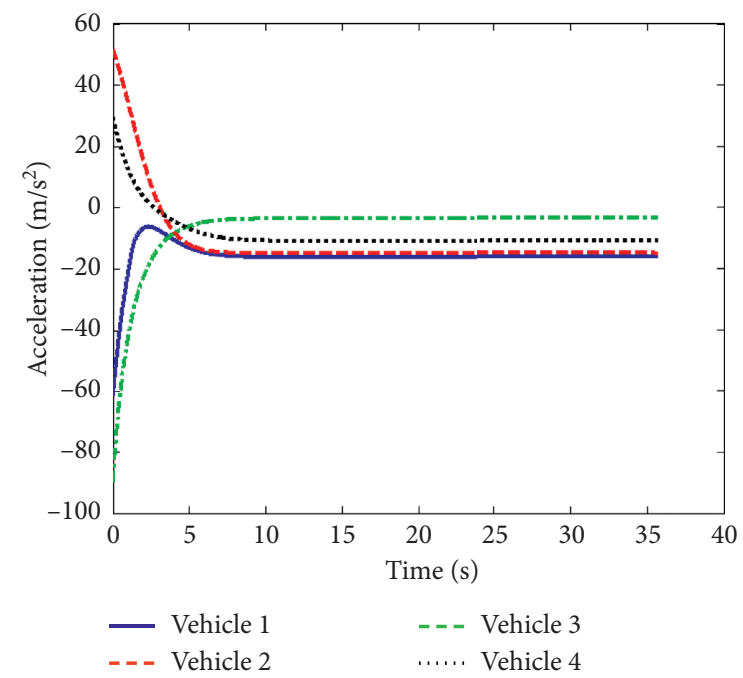

(b)

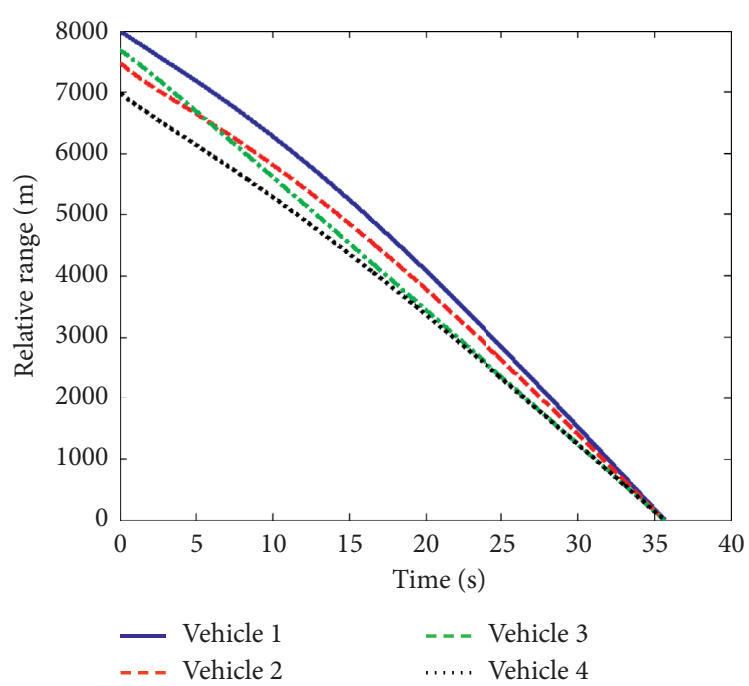

(c)

(d)

FIgURE 3: Continued. 


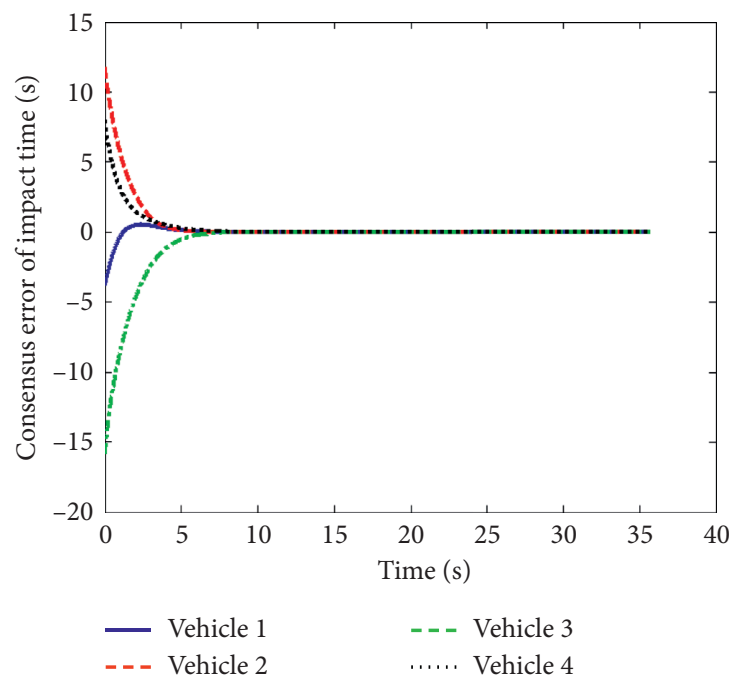

(e)

Figure 3: Simulation results under undirected and connected communication topology. (a) Vehicle trajectory. (b) Heading error. (c) Lateral acceleration. (d) Range variation. (e) Consensus error of flight time.

that the proposed guidance law can be applied in cooperative arrival for multiple vehicles.

5.2. Case 2: Leader-Follower. In this subsection, the leaderfollower communication topology between the vehicles is demonstrated in Figure 4. We consider that four vehicles arrive at a target. The detailed simulation parameters are the same as those of case 1, which are tabulated in Table 1. Vehicle 3 acts as the leader, which means that vehicle 3 cannot receive information from the other vehicles.

The Laplacian matrix of the communication topology can be acquired as

$$
\mathbf{L}=\left[\begin{array}{cccc}
2 & -1 & 0 & -1 \\
-1 & 3 & -1 & -1 \\
0 & -1 & 2 & -1 \\
0 & 0 & 0 & 0
\end{array}\right]
$$

Simulation results are demonstrated in Figure 5. Dashed line, dotted line, solid line, and dash-dotted line stand for vehicles $1,2,3$, and 4 , respectively. It can be concluded from the vehicle trajectories in Figure 5(a) that all four vehicles can arrive at the target. Furthermore, the range variation in Figure 5(d) means that all the vehicles' relative ranges converge to zero at the same time, implying that a successful simultaneous arrival is achieved under the proposed law. The variance of the heading error angles is depicted in Figure 5(b), and all of them decline to zero at the end of the engagement, which is in line with the analysis in equation (12). Vehicle 3 acts as the leader, which means its control parameter will remain constant at 1 during the homing process. The other vehicles will adjust their control parameters according to vehicle 3. After the follower vehicles reach

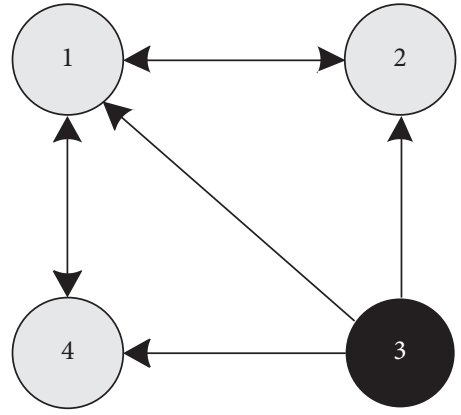

FIgURE 4: Leader-follower communication topology among vehicles.

an agreement with the leader in flight time, all the vehicles' control parameters will be 1 . This is consistent with the simulation results in Figures 5(c) and 5(e). It is obvious that the flight time of each vehicle can reach an agreement in finite time under the proposed law. This simulation proves the proposed guidance law can also be applied in cooperative arrival even if communication failures exist.

\section{Conclusion}

This paper proposes a guidance law for multiple vehicles arriving at a target cooperatively. The Lyapunov-based guidance law is proposed, and the flight time can be calculated with control parameter equal to one. Specifically, we assume that the control parameters are initialized with one at each time step. Then, by exchanging the total flight time between the vehicle and its neighbors under an undirected and connected communication topology, the control parameter will be adjusted to reduce the disparities of the flight time. After the consensus of the flight time, the control parameters will remain constant at one. The effectiveness of 

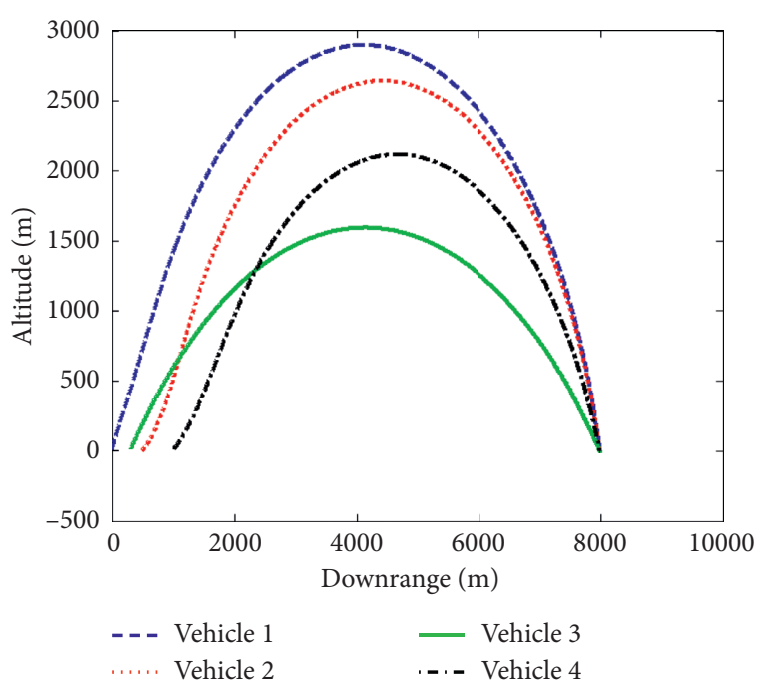

(a)

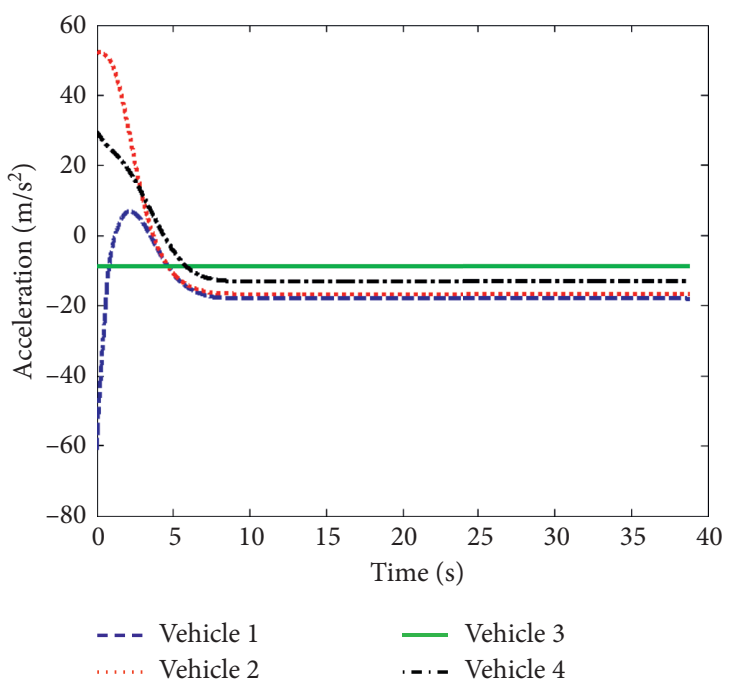

(c)

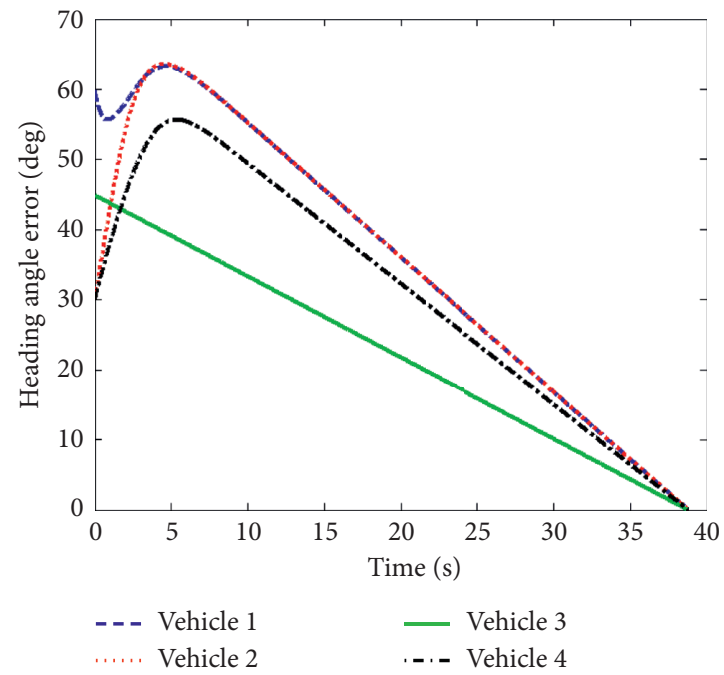

(b)

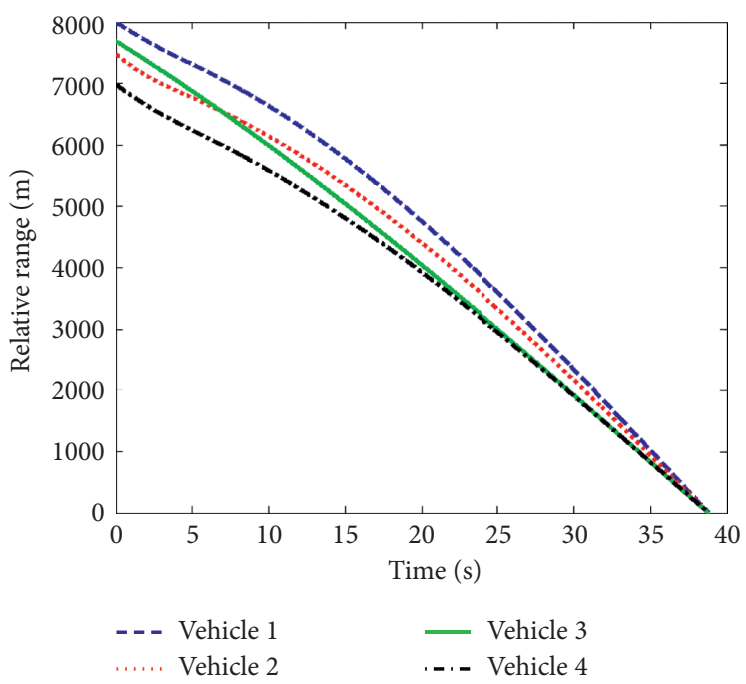

(d)

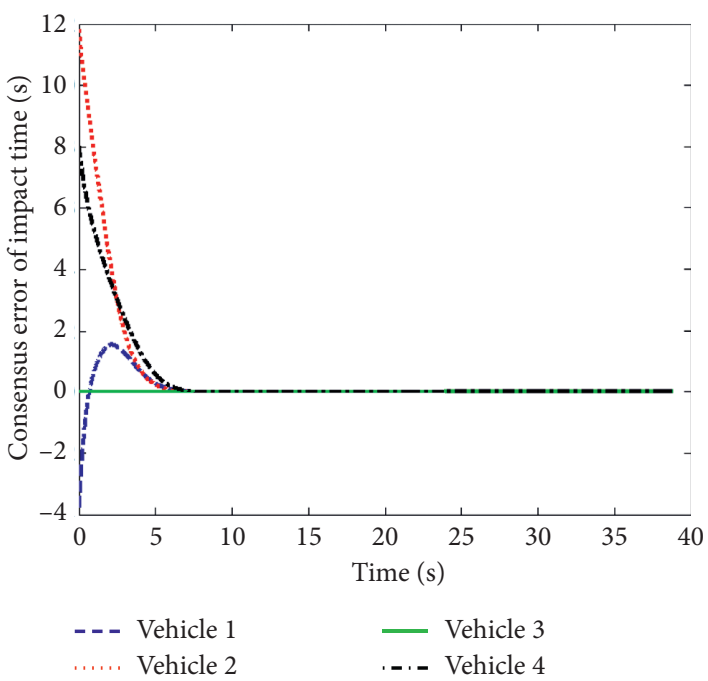

(e)

Figure 5: Simulation results under leader-follower communication topology. (a) Vehicle trajectory. (b) Heading error. (c) Lateral acceleration. (d) Range variation. (e) Consensus error of flight time. 
the proposed method is demonstrated with simulations. Compared with previous work, this paper deals with the consensus of the flight time directly rather than the estimation of time-to-go. In future related work, the tangential acceleration should be considered in the design of guidance law.

\section{Data Availability}

The data used to support the findings of this study are available from the corresponding author upon request.

\section{Conflicts of Interest}

The authors declare that they have no conflicts of interest.

\section{Acknowledgments}

This study was cosupported in part by the National Natural Science Foundation of China (nos. 61903146, 61873319, and 61803162).

\section{References}

[1] S. Ruiz, L. Guichard, N. Pilon, and K. Delcourte, "A new air traffic flow management user-driven prioritisation process for low volume operator in constraint: simulations and results," Journal of Advanced Transportation, vol. 2019, Article ID 1208279, 21 pages, 2019.

[2] K. Raghuwaiya, B. Sharma, and J. Vanualailai, "Leader-follower based locally rigid formation control," Journal of Advanced Transportation, vol. 2018, Article ID 5278565, 14 pages, 2018.

[3] S. Hao, L. Yang, L. Ding, and Y. Guo, "Distributed cooperative backpressure-based traffic light control method," Journal of Advanced Transportation, vol. 2019, Article ID 7481489, 14 pages, 2019.

[4] I. S. Jeon, J. I. Lee, and M. J. Tahk, "Impact-time-control guidance law for anti-ship missiles," IEEE Transactions on Control Systems Technology, vol. 14, no. 2, pp. 260-266, 2006.

[5] I. S. Jeon, J. I. Lee, and M. J. Tahk, "Guidance law to control impact time and angle," in Proceedings of the International Conference on Control and Automation, pp. 852-857, Hong Kong, China, March 2007.

[6] H.-G. Kim, J.-Y. Lee, H. J. Kim, H.-H. Kwon, and J.-S. Park, "Look-angle-shaping guidance law for impact angle and time control with field-of-view constraint," IEEE Transactions on Aerospace and Electronic Systems, vol. 56, no. 2, pp. 1602$1612,2020$.

[7] M. Kim, B. Jung, B. Han, S. Lee, and Y. Kim, "Lyapunov-based impact time control guidance laws against stationary targets," IEEE Transactions on Aerospace and Electronic Systems, vol. 51, no. 2, pp. 1111-1122, 2015.

[8] Z. Cheng, B. Wang, L. Liu, and Y. Wang, "A composite impact-time-control guidance law and simultaneous arrival," Aerospace Science and Technology, vol. 80, pp. 403-412, 2018.

[9] T.-H. Kim, C.-H. Lee, I.-S. Jeon, and M.-J. Tahk, "Augmented polynomial guidance with impact time and angle constraints," IEEE Transactions on Aerospace and Electronic Systems, vol. 49, no. 4, pp. 2806-2817, 2013.

[10] R. Tekin, K. S. Erer, and F. Holzapfel, "Polynomial shaping of the look angle for impact-time control," Journal of Guidance, Control, and Dynamics, vol. 40, no. 10, pp. 2668-2673, 2017.
[11] R. Tekin, K. S. Erer, and F. Holzapfel, "Adaptive impact time control via look-angle shaping under varying velocity," Journal of Guidance, Control, and Dynamics, vol. 40, no. 12, pp. 3247-3255, 2017.

[12] R. Tekin and K. S. Erer, "Impact time and angle control against moving targets with look angle shaping," Journal of Guidance, Control, and Dynamics, vol. 43, no. 5, pp. 1020-1025, 2020.

[13] M.-J. Tahk, S.-W. Shim, S.-M. Hong, H.-L. Choi, and C.-H. Lee, "Impact time control based on time-to-go prediction for sea-skimming antiship missiles," IEEE Transactions on Aerospace and Electronic Systems, vol. 54, no. 4, pp. 2043-2052, 2018.

[14] I.-S. Jeon, J.-I. Lee, and M.-J. Tahk, "Homing guidance law for cooperative attack of multiple missiles," Journal of Guidance, Control, and Dynamics, vol. 33, no. 1, pp. 275-280, 2010.

[15] J. Zhou, J. Yang, and Z. Li, "Simultaneous attack of a stationary target using multiple missiles: a consensus-based approach," Science China Information Sciences, vol. 60, no. 7, Article ID 070205, 2017.

[16] Z. Hou, L. Liu, Y. Wang, J. Huang, and H. Fan, “Terminal impact angle constraint guidance with dual sliding surfaces and model-free target acceleration estimator," IEEE Transactions on Control Systems Technology, vol. 25, no. 1, pp. 85-100, 2017.

[17] S. Wang, Y. Guo, S. Wang, Z. Liu, and S. Zhang, "Cooperative guidance considering detection configuration against target with a decoy," IEEE Access, vol. 8, pp. 66291-66303, 2020.

[18] W. Ren, R. W. Beard, and E. M. Atkins, "Information consensus in multivehicle cooperative control," IEEE Control Systems, vol. 27, no. 2, pp. 71-82, 2007.

[19] R. Olfati-Saber and R. M. Murray, "Consensus problems in networks of agents with switching topology and time-delays," IEEE Transactions on Automatic Control, vol. 49, no. 9, pp. 1520-1533, 2004.

[20] N. Zoghlami, L. Beji, and R. Mlayeh, "Finite-time consensus of networked nonlinear systems under directed graph," in Proceedings of the European Control Conference, pp. 546-551, Strasbourg, France, June 2014.

[21] D. Zhou, S. Sun, and K. L. Teo, "Guidance laws with finite time convergence," Journal of Guidance, Control, and Dynamics, vol. 32, no. 6, pp. 1838-1846, 2009. 